\title{
P300-Based Speller Brain-Computer Interface
}

\author{
Reza Fazel-Rezai \\ Department of Electrical Engineering, University of North Dakota \\ USA
}

\section{Introduction}

In this chapter, recent advances in the P300-based speller brain-computer interface (BCI) are discussed. Brain Computer Interface (BCI) technology provides a direct interface between the brain and a computer for people with severe movement impairments. The goal of $\mathrm{BCI}$ is to liberate these individuals and to enable them to perform many activities of daily living thus improving their quality of life and allowing them more independence to play a more productive role in society and to reduce social costs. A BCI involves monitoring conscious brain electrical activity, via electroencephalogram (EEG) signals, and detecting characteristics of brain signal patterns, via digital signal processing algorithms, that the user generates in order to communicate with the outside world.

A BCI system has an input, an output, and a pattern recognition algorithm that maps the input to the output (Wolpaw et al., 2002). Based on the type of input, there are four categories for a BCI system. A P300-based BCI is one of them. The focus in this chapter is the P300-based BCI. The P300 evoked potential speller was introduced by Farwell and Donchin (Farwell \& Donchin, 1988). This paradigm has been the most widely used P300-based speller and a benchmark for many research groups.

Several shortcomings of existing P300-based BCIs have been identified, and many research groups have tried to overcome those shortcomings. However, more progress in resolving many of the challenges currently experienced in P300-based speller should be made to move $\mathrm{BCI}$ into the realm of practicality and to take it outside research laboratories into practical applications.

This chapter is organized as follows. First, a brief introduction to a BCI system will be given. Then, different types of BCI systems and P300-based speller BCI will be introduced. Details of a P300-based speller BCI including signal processing methods, sensitivity parameters, habituation, applications, and different variations of the system will be explained in detail. Then, recent advances including a new P300-based speller paradigm will be presented and results will be discussed.

\section{Brain Computer Interface (BCl)}

$\mathrm{BCI}$ technology involves monitoring conscious brain electrical activity, via EEG signals, and detecting characteristics of EEG patterns, via digital signal processing algorithms, that the user generates to interact with environment. It has the potential to enable the physically 
disabled people to perform many activities. Therefore, it can improve their quality of life and productivity, allow them more independence, and reduce social costs. The challenge with $\mathrm{BCI}$, however, is to extract the relevant patterns from the EEG signals produced by the brain each second.

EEG signals (typically 64 channels) can be recorded from the scalp or brain surface using a specific system of electrode placement called the International 10-20 system (Jasper, 1957). EEG signals are voltage changes of tens of microvolts at frequencies ranging from below 1 $\mathrm{Hz}$ to about $50 \mathrm{~Hz}$. A BCI system has different components; an input, a pattern recognition algorithm, an output (Wolpaw et al., 2002). In addition, it has a protocol that determines the interaction of the $\mathrm{BCI}$ system with the user. A simple schematic diagram of a $\mathrm{BCI}$ system is shown in Figure 1.

The input of a BCI system is the EEG signal. EEG signals can be recorded invasively (i.e., intracortical) or non-invasively (i.e., surface EEG). The EEG signals can be the results of an evoked or a spontaneous signal. Evoked EEG is due to a stimulus and spontaneous EEG is a brain activity rhythm that is not the result of any stimulation. Pattern recognition components of a BCI system are the parts of the system that analyze EEG to extract a signature from EEG signals as shown in Figure 1. This includes signal processing methods for preprocessing (e.g., noise removal), feature extraction, classification, and post processing methods. Many different approaches from linear to nonlinear have been used (Mirghasemi et al., 2006). The output of a BCI system is connected to a computer, or a device that is supposed to be controlled using the BCI system. Spelling devices, i.e., virtual keyboard, is one of them. The operating protocol of a BCI system determines how the system interact with the environment, e.g., if the system supposed to work continuously or not and how the system should be turned off.

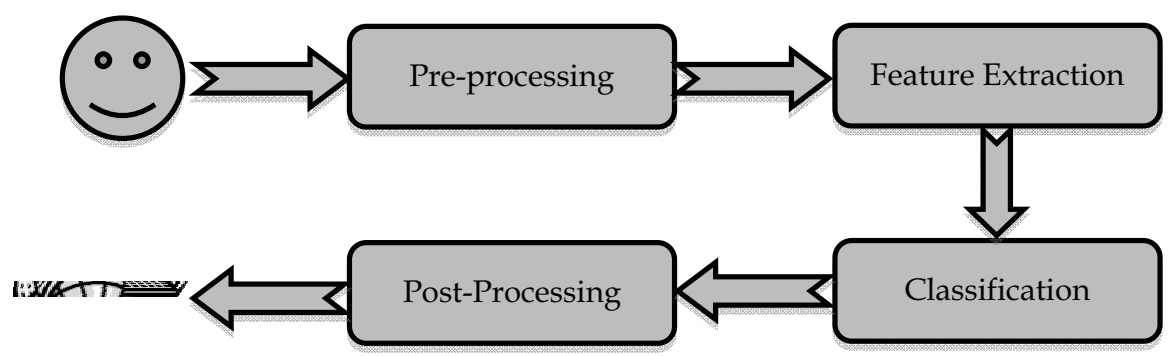

Fig. 1. A BCI system receives recorded EEG signals and extract signatures of the brain activity using several signal processing algorithm to interact with outside environment.

\section{Different Brain Computer Interface (BCl) Systems}

Based on the type of input, components, output, and protocol, BCI systems are categorized in different groups. Various properties in EEG can be used as the input of a BCI system such as rhythmic brain activity, event-related potentials, event-related desynchronization (ERD) 
and event-related synchronization (ERS). In general, four different types of brain activity reflected in EEG signals have been mostly used as the BCI system inputs: 1) visual-evoked potentials, 2) slow cortical potentials, 3) mu and beta rhythms, and 4) the P300 component of Event-Related Potentials, or ERPs) (Wolpaw et al., 1991; Blankertz et al., 2004; Pfurtscheller et al., 1993; Scherer et al., 2004; Birch \& Mason, 2000; Kostov \& Kostov, 2000).

\subsection{Rhythmic brain activity}

Normal brain waves show different rhythmic activity based on the level of consciousness. This happens not only during the sleep (different sleep stages) but it can be seen in EEG during the waking state. These rhythms are affected by internal parameters such as subject's actions and thoughts and external factors such as fatigue and stress. For example, when a subject plans to move an object (without performing the task), a particular rhythm can be generated in EEG signal. The fact that mere thoughts affect the brain rhythms can be used as the input for a BCI system. The frequency bands from low to high frequencies are called delta $(\delta)$, theta $(\theta)$, alpha $(\alpha)$, beta $(\beta)$, and gamma $(\gamma)$ as shown in Table 1 .

\begin{tabular}{|c|c|}
\hline Band & Frequency Range \\
\hline$\delta$ & $0.5-4 \mathrm{~Hz}$ \\
\hline$\theta$ & $4-7.5 \mathrm{~Hz}$ \\
\hline$\alpha$ & $8-13 \mathrm{~Hz}$ \\
\hline$\beta$ & $14-26 \mathrm{~Hz}$ \\
\hline$\gamma$ & $>30 \mathrm{~Hz}$ \\
\hline
\end{tabular}

Table 1. EEG rhythmic frequency ranges.

EEG waves below $4 \mathrm{~Hz}$ (usually $0.5-3.5 \mathrm{~Hz}$ ) belong to the delta $(\delta$ ) waves. Infants show irregular delta activity of $2-3.5 \mathrm{~Hz}$ in the waking state. However, delta waves are only seen in deep sleep in adults and they have not been used in BCI systems. Theta $(\theta)$ waves are between 4 and $7.5 \mathrm{~Hz}$. The alpha $(\alpha)$ rhythm is in the range of $8-13 \mathrm{~Hz}$ and is seen with eyes closed and under conditions of physical relaxation. $\mathrm{Mu}(8-12 \mathrm{~Hz})$ and beta $(14-26 \mathrm{~Hz})$ rhythms are mostly used in BCI systems. In people who are awake, the primary sensory or motor cortical areas often display 8-12 Hz EEG activity when they are not engaged in processing sensory input or producing motor output. This idling activity is called the mu rhythm when focused over the somatosensory or motor cortex. These mu rhythms are usually associated with $14-26 \mathrm{~Hz}$ beta rhythms. While some beta rhythms are harmonics of mu rhythms, some are separable from them by topography and/or timing, and thus are independent EEG features. It has been shown that subjects were able to raise and lower mu rhythm waves by thinking about activities such as relaxing (raising mu activity) and lifting weights (decreasing mu activity) (Pfurtscheller, 1993). In addition, it has been shown that mu rhythm is modulated by the expression of self-generated movement and observation and imagination of movement (Pineda et al., 2000).

\subsection{Event-Related Potentials (ERPs)}

Event-related potentials (ERPs) are changes in the EEG that occur in response to a stimulus. ERP is a voltage fluctuation in the EEG induced within the brain that is time-locked to a sensory, motor, or cognitive event. The stimulus can be visual, auditory, or somatosensory. 
ERPs provide a different indication of how the stimulus is processed. They are measures to reflect the responses of the brain to events in the external or internal environment of the organism. Although they are not easy to be detected, they have wide usage for clinicaldiagnostic and research purposes. It should be noted that whereas the amplitude of ongoing EEG activity is in the range of 50 microvolts, the amplitude of the ERP is much smaller in the range of 5 to 10 microvolts. The "peaks" and "valleys" in the ERP are termed components, with positive deflections labelled with " $\mathrm{P}$ " and negative deflections labelled with " $\mathrm{N}$ ". The N400, for example, is the negative peak near 400 milliseconds after a stimulus onset. The P300 component is the fourth major positive peak. The P300 component of ERP was first reported by Sutton (Sutton et al., 1965). The P300 component of ERP is a positive peak at about $300 \mathrm{~ms}$ after stimulus. It is elicited with a simple, two-stimulus discrimination task. This procedure has been dubbed the "oddball paradigm", whereby two stimuli are presented in a random series such that one of them (the "oddball") occurs relatively infrequently. A user is asked to distinguish between the stimuli by mentally noting each oddball stimulus, which creates a P300 wave, and ignoring the standard stimulus. Unfortunately, the P300 has a relatively small amplitude (5-10 microvolts), and cannot be readily seen in an EEG signal (10-100 microvolts). The averaging process is used to decrease the influence of the background EEG, but it typically takes 30 to 50 trials before the contribution of the background EEG subsides and a good, clean representation of the P300 is obtained.

\section{P300-BCI Speller}

The most widely used P300-based BCI is a spelling device designed by Farwell and Donchin in 1988 (Farwell \& Donchin, 1988). Since then, it has been a benchmark for P300-based BCI systems in the research laboratory. In this speller, a matrix of six by six symbols, comprising all 26 letters of the alphabet and 10 digits (0-9), is presented to the user on a computer screen (Figure 2). The rows and columns of the matrix are intensified successively for $100 \mathrm{~ms}$ in a random order. After intensification of a row/column, the matrix is blank for $75 \mathrm{~ms}$. At any given moment, the user focuses on the symbol he/she wishes to communicate, and mentally counts the number of times the selected symbol flashes. In response to the counting of this oddball stimulus, the row and column of the selected symbol elicit a P300 wave, while the other 10 rows and columns do not. Detection of the P300 makes it possible to match the responses to one of the rows and one of the columns, and thus identify the target symbol.

$$
\begin{array}{cccccc}
\text { A } & \text { B } & \text { C } & \text { D } & \text { E } & \text { F } \\
\text { G } & \text { H } & \text { I } & \text { J } & \text { K } & \text { L } \\
\text { M } & \text { N } & \text { O } & \text { P } & \text { Q } & \text { R } \\
\text { S } & \text { T } & \text { U } & \text { V } & \text { W } & \text { X } \\
\text { Y } & \text { Z } & 1 & 2 & 3 & 4 \\
5 & 6 & 7 & 8 & 9 & 0
\end{array}
$$

Fig. 2. The P300-based BCI speller originally introduced by Farwell and Donchin (Farwell \& Donchin, 1988). 
One of the greatest advantages of the $\mathrm{P} 300$-based $\mathrm{BCI}$ is that it does not require intensive user training, as P300 is one of the brain's "built-in" functions. However, P300 detection for real-time applications is not easy. Although signal-processing methods play a crucial role in $\mathrm{BCI}$ design, they cannot solve every problem. While they can enhance the signal-to-noise ratio, extract proper features, and classify them into different groups, they cannot directly address the impact of changes in the signal itself. There are several issues to be addressed before any P300-based BCI can be taken outside the research laboratory and put to practical application:

A) Transfer rate: Typically, many channels and several features should be used. In addition, the ensemble averaging of a large number of trials is required to improve the signal-to-noise ratio, because the P300 is buried in the ongoing EEG (Donchin et al., 2000).

B) Accuracy: For real-time applications, the P300-based BCI accuracy should be very high to be reliable to be used in real-world environment.

C) EEG pattern variability: EEG signal patterns change due to factors such as motivation, frustration, level of attention, fatigue, mental state, learning, and other nonstationarities that exist in the brain. In addition, different users might provide different EEG patterns.

\subsection{Recent Advances in the P300-BCI Speller}

Recently, many research groups have been trying to increase both transfer rate and accuracy, which are interdependent (Serby et al., 2005). The following parameters have to be addressed by a BCI system:

(a) Attentional blink: Attentional blink occurs if the interval between two targets is less than 500ms (Raymond et al., 1992). In such a case, the first target is correctly identified, while the second is not detected at all. This can be a source of P300 speller error if a non-target row/column near the target attracts attention by flashing less than $500 \mathrm{~ms}$ before the target is flashed.

(b) Repetition blindness: If two identical targets in a stream of non-targets are flashed at intervals of less than 500ms, the second target may be missed (Kanwisher, 1987). This can be a source of error whenever a target row (column) is flashed less than 500ms after flashing a target column (row). Although they are intensified in random sequences, because the total intensification plus blank time is $175 \mathrm{~ms}$, there are several cases where the interval between two targets is less than $500 \mathrm{~ms}$. This is another source of error.

(c) Target probability: It has been shown that the P300 amplitude is related to the probability of oddball occurrence (target row/column flashing) (Donchin et al., 2000). The less probable the oddball event, the larger the P300 amplitude. Although, in this paradigm, the probability of creating the P300 wave is 0.17 (2/12), the P300 amplitude can be increased by decreasing the oddball probability.

(d) Habituation: Attention decreases with repeated presentation of the same stimulus. When the user loses focus on the target character, the P300 is not elicited, and the system error increases with time.

(e) Row/Column error sensitivity: In the P300-based speller, the target symbol is the intersection of the target row and column, both of which, therefore, have to be detected correctly. This creates a sensitive system to error in detecting target row and column.

Fazel-Rezai (Fazel-Rezai, 2007) performed an analysis to reveal potential human error in the P300-based BCI system. In this work, two data sets were used. The first dataset was from BCI 2003 competition provided by Blankertz (Blankertz, et al, 2004), Wadsworth Center, 
Albany, NY. This dataset was selected, because the results can be compared with the results of other works and repeated by other research groups. Features were extracted from averaged Mexican hat wavelet coefficients. More details of the feature extraction can be found in (Fazel-Rezai \& Peters, 2005; Fazel-Rezai \& Ramanna, 2005; Ramanna \& Fazel-Rezai, 2006). It should be noted that since the objective of analysis was to reveal the possible errors and compare speller paradigms, no learning set was used.

In the first data set, BCI competition (Blankertz, et al, 2004), EEG signals were recorded from 64 electrodes; however, they used only $\mathrm{Fz}, \mathrm{Pz}, \mathrm{Cz}, \mathrm{C} 1$, and $\mathrm{C} 2$ channels. Row/column intensifications $(100 \mathrm{msec})$ were block randomized in blocks of 12 . Sets of 12 intensifications were repeated 15 times for each character. Each sequence of 15 sets of intensifications was followed by a $2.5 \mathrm{~s}$ period, and during this time the matrix was blank. This period informed the user that this character was completed and to focus on the next character in the word that was displayed on the top of the screen. In other words, for each character, 180 entries of feature values are stored 90 for row intensification and 90 for column intensification. For example, " $A$ " is recognized only when row 7 and column 1 features indicate a P300 component. The signals were digitized at $240 \mathrm{~Hz}$ and collected from one subject in two sessions. Each session consisted of a number of runs. In each run, the subject focused attention on a series of characters. Target words presented to the subject were: BOWL, CAT, DOG, FISH, FISH, GLOVE, HAT, HAT, RAT, SHOES, and WATER. Note that words FISH and HAT were presented two times. The total number of target characters in these words is 42. The results of analysis is shown in Table 1.

\begin{tabular}{|c|c|c|c|c|c|c|c|c|c|c|c|c|c|c|c|c|c|c|c|c|c|c|c|}
\hline Word & \multicolumn{3}{|c|}{ BOWL } & \multicolumn{3}{|c|}{ CAT } & \multicolumn{3}{|c|}{ DOG } & \multicolumn{5}{c|}{ FISH } & \multicolumn{5}{c|}{ FISH } & \multicolumn{5}{c|}{ GLOVE } \\
\hline Target & B & O & W & L & C & A & T & D & O & G & F & I & S & H & F & I & S & H & G & L & O & V & E \\
\hline Detected & B & O & W & F & C & A & T & D & O & G & F & H & M & H & E & D & S & A & G & H & 7 & V & E \\
\hline E(Col) & 0 & 0 & 0 & 0 & 0 & 0 & 0 & 0 & 0 & 0 & 0 & -1 & 0 & 0 & -1 & 1 & 0 & -1 & 0 & -4 & 0 & 0 & 0 \\
\hline E(Row ) & 0 & 0 & 0 & -1 & 0 & 0 & 0 & 0 & 0 & 0 & 0 & 0 & -1 & 0 & 0 & -1 & 0 & -1 & 0 & 0 & 3 & 0 & 0 \\
\hline
\end{tabular}

\begin{tabular}{|c|c|c|c|c|c|c|c|c|c|c|c|c|c|c|c|c|c|c|c|}
\hline Word & \multicolumn{3}{|c|}{ HAT } & \multicolumn{3}{|c|}{ HAT } & \multicolumn{3}{|c|}{ RAT } & \multicolumn{4}{c|}{ SHOES } & \multicolumn{5}{c|}{ WATER } \\
\hline Target & H & A & T & H & A & T & R & A & T & S & H & O & E & S & W & A & T & E & R \\
\hline Detected & H & B & T & L & A & T & R & A & Z & S & H & A & Q & M & S & A & T & E & R \\
\hline E(Col) & 0 & 1 & 0 & 4 & 0 & 0 & 0 & 0 & 0 & 0 & 0 & -2 & 0 & 0 & -4 & 0 & 0 & 0 & 0 \\
\hline E(Row ) & 0 & 0 & 0 & 0 & 0 & 0 & 0 & 0 & 1 & 0 & 0 & -2 & 2 & -1 & 0 & 0 & 0 & 0 & 0 \\
\hline
\end{tabular}

Table 1. Target character and detected character in the target words presented to subject. The shaded cells show when an error occurred. Differences between column/row of the target and that of the detected characters are shown in the fourth/fifth row of the table, respectively. 
The first and second rows in Table 1 show the target words and characters for the first data sets, respectively. The detected character is shown in the third row. Differences between detected columns/rows and target columns/rows are shown in the fourth and fifth rows of the table. If the target row or column is detected correctly, zero is shown. If the detected row is at the bottom or top of the target row, a positive or negative number is shown respectively. In a similar notation, if the detected column is at the right or left of the target column a positive or negative number is shown, respectively. Shaded cells in this table show if there is an error in detection. From total 42 target characters, 27 characters $(65 \%)$ were detected correctly and 15 characters (35\%), shaded in the table, were not detected correctly because their row or column (or both) was identified wrongly. Since no training set was used, low accuracy results were expected. In this study, error cases were analyzed to search for a consistent pattern for the error.

Figure 3 shows another representation of the row and column differences. The focus in this representation is how close the detected character is to the target character. It shows that 27 times target character was detected correctly. However, any other nonzero number shows an error in detection (they are shaded cells). In Figure 3, for example, number "3", located on the top of " 27 ", indicates that "three times" the detected row was one row above target row while the column was detected correctly or number " 2 ", located at the far left in the figure and four cells away from 27, indicates that "two times" a column was detected which was on the left side of target column and four columns away from it, while the row was detected correctly.

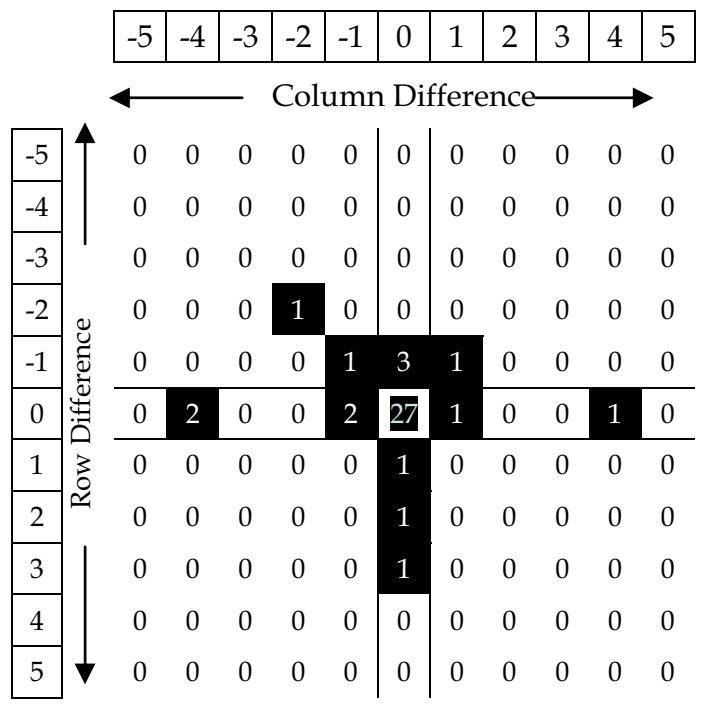

Fig. 3. Error in detecting target row and column as a function of the distance between target and detected row and column. In this figure the target row/column is considered at the center. The shaded cells indicate when an error occurs and the value of the cells show the difference between target and detected row or column. 


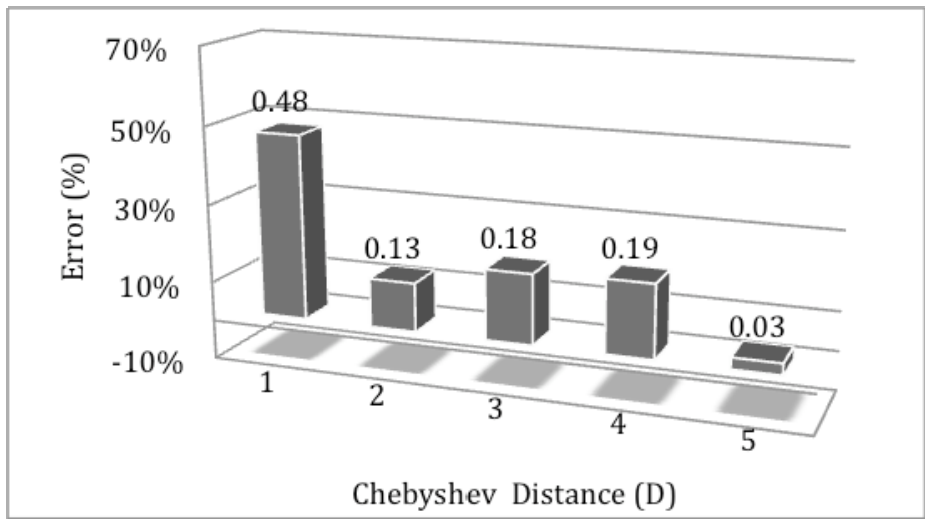

Fig. 4. Percentage of error for Farwell-Donchin paradigm as a function of distance between target (center cell) and detected row/column for both data sets. The Chebyshev distance was considered.

Using the Chebyshev distance, the distance between detected row/column and target row/column was calculated as follows:

$$
={ }_{\rightarrow \infty}\left(\sum_{=} \mid-1\right)^{\prime}=(-1)
$$

where and represent the locations of the target (center) and the wrongly detected characters, respectively. Percentage of the error cases for different values of D is shown in Figure 4.

It can be concluded that the majority of the error cases in detecting a character are when the adjacent row/column to the target row/column ( $D=1)$ was detected $(48 \%)$. This can be attributed to subject's attention where the subject is unable to focus precisely only on the target letter and flashing of the adjacent row/column elicits the P300. It confirms the hypothesis that intensification of adjacent rows and columns to the desired character creates unwanted P300.

\subsection{Region Based P300-Basd BCI}

To overcome the problem described in the pervious section and several other shortcomings, an innovative P300-based speller, in which the screen is divided into seven regions (Figure 5) was proposed (Fazel-Rezai, 2008). In addition to 26 alphabet letters and 10 digits (0-9), 13 special characters $(@, \# \%, *, /, \ldots)$ are included, for a total of 49 symbols. The target symbol is recognized at two levels. First, the 49 symbols are placed in seven regions (seven symbols per region), and the regions are flashed in a random sequence. The user focuses on the region with the target symbol. Second, after the target region has been detected using the P300 wave, the seven symbols in the target region will be distributed among the seven regions (one symbol per region). Flashing these seven regions again will enable the identification of the target symbol. The challenges mentioned in the pervious section (a-e) were addressed as follows: 
(a) Attentional blink: In the region-based system, the regions can be widely spaced on the screen, to reduce the error caused by attentional blink.

(b) Repetition blindness: Although the regions are intensified in random order, the time between intensifications in a region will be set for more than $500 \mathrm{~ms}$, to eliminate the repetition blindness error.

(c) Target probability: The probability of the oddball in this paradigm is $14 \%(1 / 7)$ - less than that for the Farwell and Donchin paradigm. Lower oddball probability increases P300 amplitude, and has two benefits; it decreases the number of trials required for each character, thereby increasing the transfer rate, and it makes P300 detection easier, which increases the accuracy.

(d) Habituation: Habituation in the new speller can be reduced by changing the region locations on the screen, their background, and other visual effects that create a novelty in the paradigm. This novelty increases the user's attention for the later presentation of a new symbol stimulus.

(e) Row/column error sensitivity: This speller has no row/column error sensitivity. Although it has a two-level error sensitivity, a "backup" word can be displayed (as the eighth region) and flashed at the beginning of the second level. If the region is not detected correctly at the first level, the user can go back to the first level by focusing on a region that is considered for "backup" (not shown in Figure 5).

Furthermore, the new region-based P300 BCI speller has several new features:

(f) Number of symbols: The total number of symbols has increased from 36 to 49 .

(g) User selection: The user can choose several parameters, including region locations, region shapes, and background colors.

(h) Adaptive detection: This is one of the most important features of the proposed P300based BCI. The objective is to design a system which adapts to the user's performance. In general, when a user has significant response (e.g., when the P300 amplitude is high), fewer trial repetitions, channels, and features are needed for a correct decision (P300 detection).

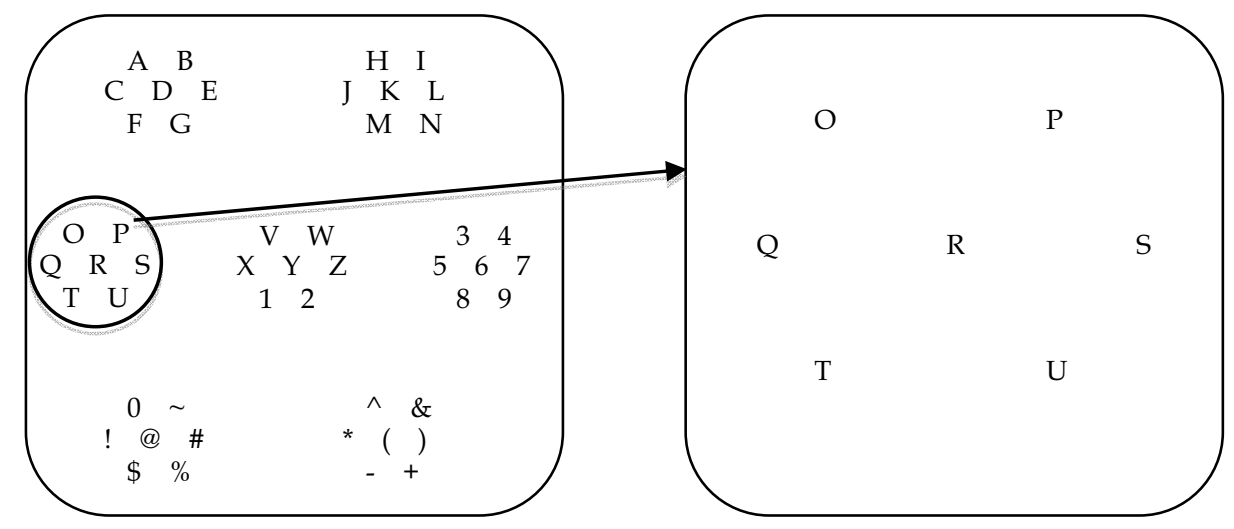

(a)

(b)

Fig. 5. The region-based paradigm. a) The first level of intensification, each group contains up to 7 characters. b) One of the regions is expanded in the second level. 
To evaluate the new paradigm, a data set was recorded from 10 subjects (20-29 years, 2 females). Before each experiment the procedure was explained to the subjects and they signed the informed consent approved by the Ethics Board. Subjects were seated on a chair in front of the screen and asked to be relaxed and avoid moving as much as possible during the experiments. Two paradigms were presented to all subjects. They were asked to spell "P3A". Therefore, EEG signals for total of 30 characters for each paradigm were collected. They were recorded across midline recording sites of $\mathrm{Fz}, \mathrm{Cz}$, and $\mathrm{Pz}$ referenced to the left mastoid with a forehead ground. EEG signals were sampled at $500 \mathrm{~Hz}$.

The results for the new data set and for the Farwell-Donchin paradigm and the new paradigm are shown in Table 2 and Table 3, respectively (Abhari \& Fazel-Rezai, 2006). Table 2 shows in 22 error cases out of 60 cases. However, this error was reduced to 11 out of 60 cases in a region-based paradigm (Table 3 ), that is $50 \%$ improvement in the accuracy.

\begin{tabular}{|c|c|c|c|c|c|c|c|}
\hline Target character $\rightarrow$ & & & & & & & \multirow{3}{*}{ Total Error } \\
\hline Target column/row $\rightarrow$ & 4 & 3 & 5 & 5 & 1 & 1 & \\
\hline Subject & \multicolumn{6}{|c|}{ Detected column/row } & \\
\hline 1 & 4 & 3 & 5 & 5 & 5 & 4 & 2 \\
\hline 2 & 1 & 3 & 6 & 5 & 1 & 1 & 2 \\
\hline 3 & 4 & 5 & 1 & 1 & 1 & 1 & 3 \\
\hline 4 & 4 & 3 & 1 & 5 & 1 & 1 & 1 \\
\hline 5 & 3 & 4 & 5 & 5 & 1 & 1 & 2 \\
\hline 6 & 6 & 3 & 2 & 5 & 1 & 2 & 3 \\
\hline 7 & 5 & 2 & 5 & 5 & 6 & 1 & 3 \\
\hline 8 & 1 & 3 & 6 & 5 & 1 & 4 & 3 \\
\hline 9 & 4 & 3 & 5 & 5 & 4 & 4 & 2 \\
\hline 10 & 4 & 3 & 4 & 5 & 1 & 1 & 1 \\
\hline Total Error & 5 & 3 & 6 & 1 & 3 & 4 & 22 \\
\hline
\end{tabular}

Table 2. Detected and target regions in spelling "P3A" using Donchin-Farwell paradigm.

\begin{tabular}{|c|c|c|c|c|c|c|c|}
\hline Target character $\rightarrow$ & & & & & & & \multirow{3}{*}{ Total Error } \\
\hline Target region $\rightarrow$ & 3 & 2 & 5 & 1 & 1 & 1 & \\
\hline Subject & \multicolumn{6}{|c|}{ Detected region } & \\
\hline 1 & 4 & 5 & 5 & 1 & 1 & 1 & 2 \\
\hline 2 & 3 & 3 & 5 & 1 & 1 & 1 & 1 \\
\hline 3 & 3 & 6 & 5 & 1 & 1 & 1 & 1 \\
\hline 4 & 3 & 2 & 5 & 1 & 1 & 1 & 0 \\
\hline 5 & 3 & 2 & 1 & 1 & 1 & 1 & 1 \\
\hline 6 & 1 & 2 & 5 & 1 & 1 & 1 & 1 \\
\hline 7 & 3 & 2 & 4 & 1 & 2 & 1 & 2 \\
\hline 8 & 3 & 4 & 4 & 1 & 1 & 1 & 2 \\
\hline 9 & 3 & 2 & 5 & 1 & 1 & 1 & 0 \\
\hline 10 & 3 & 2 & 1 & 1 & 1 & 1 & 1 \\
\hline Total Error & 2 & 4 & 4 & $\overline{0}$ & 1 & 0 & 11 \\
\hline
\end{tabular}

Table 3. Detected and target regions in spelling "P3A" using 7-region paradigm. 


\section{Conclusion}

In this chapter, different components and types of BCI systems were discussed briefly and P300-based BCI was discussed in more details. In order to reveal the possible sources of error in the P300-BCI paradigm, an analysis was presented. It showed that in the FarwellDonchin paradigm, the majority of error cases happened when a row/column adjacent to the target row/column is detected. Therefore, in the design of any new P300 speller paradigm this perceptual error should be considered. Then, a new paradigm which is based on dividing computer screen to different regions was presented. Experimental results of two paradigms confirmed that the accuracy in the region-based paradigm could be increased. In addition to higher accuracy, the 7-region paradigm has several advantages. 1) The number of characters has been increased from 36 to 49 . This gives more flexibility to subjects in spelling a word. 2) The oddball probability in flashing a row/column in Farwell-Donchin is $2 / 12$ or $1 / 6$. This is reduced to $1 / 7$ in the new paradigm. It has been shown that the lower probability in oddball paradigm results in higher amplitude in the P300 (Donchin et al., 2000). Therefore, in the new paradigm, P300 amplitude is larger and the detection of the P300 would be easier. This means that smaller number of flashing is required for a successful P300 detection that results in a faster P300 speller.

\section{References}

Wolpaw, J.R.; Birbaumer, N.; McFarland, D.J.; Pfurtscheller, G. \& Vaughan, T.M. (2002). Brain-computer interfaces for communication and control. Clinical Neurophysiology, Vol. 113, pp. 767-791.

Jasper, H.H. (1957). The ten-twenty electrode system of the international federation. Electroencephalogram and Clinical Neurophysiology, Vol. 10, pp. 371-375.

Wolpaw, J.R.; McFarland, D.J.; Neat, G.W. \& Forneris, C.A. (1991). An EEG-based braincomputer interface for cursor control. Electroenceph Clin Neurophys, Vol. 78, pp. 252259.

Blankertz, B.; Müller K.R.; Curio G.; Vaughan T.M.; Schalk G.; Wolpaw J.R.; Schlögl A.; Neuper C.; Pfurtscheller G.; Hinterberger T. Schröder M. \& Birbaumer N. (2004). The BCI competition 2003: progress and perspectives in detection and discrimination of EEG single trials. IEEE Trans. Biomed. Eng., Vol. 51, pp. 1044-1052.

Pfurtscheller, G.; Flotzinger, D. \& Kalcher, J. (1993). Brain computer interface-a new communication device for handicapped persons. J. of Microcomputer Applications, Vol. 16, pp. 293-299.

Scherer, R.; Müller G.R.; Neuper C.; Graimann B. \& Pfurtscheller G. (2004). An asynchronously controlled EEG-based virtual keyboard: improvement of the spelling rate. IEEE Trans Biomed. Eng., Vol. 51, No. 6, pp. 979-985.

Birch, G.E. \& Mason, S.G. (2000). Brain-computer interface research at the Neil Squire Foundation. IEEE Trans. Rehab. Eng., Vol. 8, No. 2, pp. 193-195.

Kostov, A. \& Kostov, M. (2000). Parallel man-machine training in development of EEGbased cursor control. IEEE Trans. Rehab. Eng., Vol. 8, pp. 203-205.

Sutton, S.; Braren, M.; Zubin, J. \& John E.R. (1965). Information delivery and the sensory evoked potential," Science, Vol. 155, pp. 1436-1439. 
Farwell, L.A. \& Donchin, E. (1988). Talking off the top of the head: toward a mental prosthesis utilizing event-related brain potentials. Electroenceph. Clin. Neurophysiol., pp. 510-523.

Donchin, E.; Spencer, K.M. \& Wijesinge, R. (2000). The mental prosthesis: Assessing the speed of a P300-based brain-computer interface. IEEE Trans. Rehab. Eng., Vol. 8, pp. 174-179.

Fazel-Rezai, R. \& Peters, J.F. (2005), P300 wave feature extraction: preliminary results. Proceedings of the Canadian Conference on Electrical and Computer Engineering, pp. 376379.

Fazel-Rezai, R. \& Ramanna, S. (2005). Brain signals: feature extraction and classification using rough set methods, In: Rough sets, fuzzy sets, data mining and granular computing, D. Slezak et al. (Eds), pp. 709-718, Vol. 2, Lecture Notes in Artificial Intelligence, Springer-Verlag.

Fazel Rezai, R. \& Abhari, K. (2008). A comparison between a matrix-based and a regionbased P300 speller paradigms for brain-computer interface. Proceedings of the 29th Annual International Conference of the IEEE Engineering in Medicine and Biology Society, pp. 1147-1150, Vancouver, Canada.

Fazel-Rezai, R. (2007). Human error in P300 speller paradigm for brain-computer interface. Proceedings of the 29th Annual International Conference of the IEEE Engineering in Medicine and Biology Society, pp. 2516-2519, Lyon, France.

Ramanna, S. \& Fazel-Rezai, R. (2006). A robust P300 detection based on rough sets Transactions on Rough Sets, vol. 5, pp. 207-223.

Mirghasemi, H.; Shamsollahi, M.B. \& Fazel-Rezai, R. (2006). Analysis of classifiers in P300 recognition. Proceedings of the 28th Annual International Conference of the IEEE Engineering in Medicine and Biology Society, pp. 6205-6208, New York, USA.

Mirghasemi, H., Fazel-Rezai R. \& Shamsollahi, M.B. (2006). Assessment of preprocessing methods on classifiers used in the P300 speller paradigm. Proceedings of the 28th Annual International Conference of the IEEE Engineering in Medicine and Biology Society, pp. 1319-1322, New York, USA.

Abhari, K. \& Fazel-Rezai, R. (2006). P300-based speller paradigms for brain-computer interface. Proceedings of the 29th Canadian Medical and Biological Engineering Conference, Vancouver, Canada.

Raymond, J.E. et al. (1992). Temporary suppression of visual processing in a RVSP task: An attentional blink? J. of Experimental Psychology: Human Perception and Performance, pp. 849-860.

Kanwisher, N.G. (1987). Repetition blindness: Type recognition without token individuation. Cognition, Vol. 7, pp.117-143.

Pineda, J.A; Allison B.Z. \& Vankov A. (2000). The effects of self-movement, observation, and imagination on $\mu$ rhythms and readiness potentials (RP's): Toward a braincomputer interface (BCI). IEEE Transactions on Rehabilitation Engineering, Vol.8, No. 2, pp.219-222.

Serby, H.; Yom-Tov, E. \& Inbar, G.F. (2005). An improved P300-based brain-computer interface. IEEE Trans Neural System Rehabilitation Eng. Vol. 13, No. 1, pp. 89-98.

Seller E.W. \& Donchin E. (2006). A P300-based brain-computer interface: Initial tests by ALS patients. Clinical Neurophysiology, Vol. 117, pp. 538-548. 


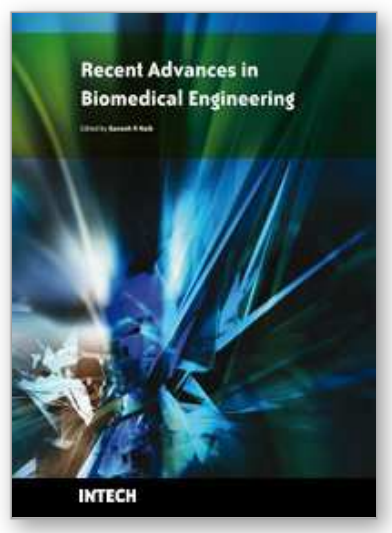

\author{
Recent Advances in Biomedical Engineering \\ Edited by Ganesh R Naik
}

ISBN 978-953-307-004-9

Hard cover, 660 pages

Publisher InTech

Published online 01, October, 2009

Published in print edition October, 2009

The field of biomedical engineering has expanded markedly in the past ten years. This growth is supported by advances in biological science, which have created new opportunities for development of tools for diagnosis and therapy for human disease. The discipline focuses both on development of new biomaterials, analytical methodologies and on the application of concepts drawn from engineering, computing, mathematics, chemical and physical sciences to advance biomedical knowledge while improving the effectiveness and delivery of clinical medicine. Biomedical engineering now encompasses a range of fields of specialization including bioinstrumentation, bioimaging, biomechanics, biomaterials, and biomolecular engineering. Biomedical engineering covers recent advances in the growing field of biomedical technology, instrumentation, and administration. Contributions focus on theoretical and practical problems associated with the development of medical technology; the introduction of new engineering methods into public health; hospitals and patient care; the improvement of diagnosis and therapy; and biomedical information storage and retrieval. The book is directed at engineering students in their final year of undergraduate studies or in their graduate studies. Most undergraduate students majoring in biomedical engineering are faced with a decision, early in their program of study, regarding the field in which they would like to specialize. Each chosen specialty has a specific set of course requirements and is supplemented by wise selection of elective and supporting coursework. Also, many young students of biomedical engineering use independent research projects as a source of inspiration and preparation but have difficulty identifying research areas that are right for them. Therefore, a second goal of this book is to link knowledge of basic science and engineering to fields of specialization and current research. The editor would like to thank the authors, who have committed so much effort to the publication of this work.

\title{
How to reference
}

In order to correctly reference this scholarly work, feel free to copy and paste the following:

Reza Fazel-Rezai (2009). P300-Based Speller Brain-Computer Interface, Recent Advances in Biomedical Engineering, Ganesh R Naik (Ed.), ISBN: 978-953-307-004-9, InTech, Available from:

http://www.intechopen.com/books/recent-advances-in-biomedical-engineering/p300-based-speller-braincomputer-interface

\section{INTECH}

open science | open minds

\section{InTech Europe}

University Campus STeP Ri

Slavka Krautzeka 83/A

\section{InTech China}

Unit 405, Office Block, Hotel Equatorial Shanghai

No.65, Yan An Road (West), Shanghai, 200040, China 
51000 Rijeka, Croatia

Phone: +385 (51) 770447

Fax: +385 (51) 686166

www.intechopen.com
中国上海市延安西路65号上海国际贵都大饭店办公楼405单元

Phone: +86-21-62489820

Fax: $+86-21-62489821$ 
(C) 2009 The Author(s). Licensee IntechOpen. This chapter is distributed under the terms of the Creative Commons Attribution-NonCommercial-ShareAlike-3.0 License, which permits use, distribution and reproduction for non-commercial purposes, provided the original is properly cited and derivative works building on this content are distributed under the same license. 